\title{
Über den Rippentiefstand ${ }^{1}$ ).
}

Von G. Ledderhose in Straßburg i. Els.

Das Wesen der ankylosierenden Erkrankungen der Wirbelsäule ist durch eine große Anzahl anatomischer und röntgenologischer Untersuchungen in fast erschöpfender Weise klargestellt worden, dagegen ist es noch nicht genügend bekannt, wie sich die auf diesen Wegen festgestellten Veränderungen im klinischen Bilde widerspiegeln. Ich habe seit längerer Zeit meine Aufmerksamkeit einem Symptom zugewandt, das bei verschiedenen pathologischen Zuständen der Wirbelsäule zutage tritt, aber bisher kaum speziell berücksichtigt wurde, nämlich die abnorme Annäherung der untersten Rippen an den Beckenka mm, die ich kurz mit $R$ ippentiefstand bezeichne. Je nach der Dicke und Konsistenz der lumbalen Weichteile sind bekanntlich die untersten Rippen verschieden deutlich abzutasten, und die I2. Rippe ist meist nicht zu fühlen. Daher ist es nicht möglich, den Grad des Rippentiefstandes exakt $z u$ bestimmen. Es genügt aber für unsere Zwecke, am stehenden Patienten festzustellen, wieviel Querfingerbreiten sich in der Scapularlinie zwischen II. Rippe und Beckenkamm eindrücken lassen. Dieser Abstand beträgt bei groß gewachsenen, jugend. lichen Individuen mit normaler Wirbelsäule e t w a vier F in ger breiten, unter pathologischen Bedingungen kann er aber vollkommen aufgehoben sein, so daB die untersten Rippen die Crista ilei berühren oder auch über sie hinaus in das Becken eingetreten sind. Zwischen diesen Extremen liegt die Summe der physiologischen und pathologischen Grade des Rippenstandes bzw. Rippentiefstandes.

Bekannt ist es, daß. bei Skoliosen und Kyphosen sich die untersten Rippen in abnormer Weise dem Becken nähern Vortrag.

1) Nach einem auf der Naturforscherversammlung in Karlsruhe gehaltenen 
und tief in dieses sich senken können, was dann nicht selten erhebliche Beschwerden verursacht. Von diesen Erkrankungen der- Wirbelsäule als Ursache des Rippentiefstandes soll aber nachstehend nicht gehandelt werden; uns interessiert das fragliche Symptom vielmehr besonders als Ausdruck der eingetretenen Verkürzung der Lendenwirbelsäule, wie sie so oft bei der Spondylitis deformans beobachtet wird, einer Erkrankung, deren Häufigkeit und Bedeutung meist unterschätzt wird.

Wenn wir allgemein die Frage aufwerfen, durch welche pathologischen Prozesse, abgesehen von Frakturen und hochgradigen Verkrümmungen, eine Verkürzung der Lendenwirbelsäule mit Rippentiefstand zustande kommen kann, so werden wir in erster Linie an eine Höhenverminderung der Bandscheiben oder auch der Wirbelkörper selbst zu denken haben. Diese Veränderungen sind aber gerade für die Spondylitis deformans bezeichnend. Sie gehört in mäßiger Ausbildung zu den ,allerhäufigsten Vorkommnissen“ (S i m monds ${ }^{1}$ ) und hat ihre Ursache in primärer Degeneration der Bandscheiben, wie sie im höheren Alter oder als Folge von Arteriosklerose, wohl auch bei regelmäßigem Arbeiten in gebückter Haltung und vielleicht auch als Inaktivitätsatrophie nach langem Nichtgebrauch (Beneke $\left.{ }^{2}\right)$ ) beobachtet wird. Anfangs sind die Bandscheiben durch rauchgraue Farbe, eine eigenartige „Verwerfung“ der Schichten sowie durch Rupturen ausgezeichnet, später tritt breiartige Erweichung ein mit zunchmender Resorption und vollständigem Verschwinden der den Bandscheiben eigentümlichen Elastizität. Dadurch treten diese dauernd über die normale Grenze der Wirbelkörper hinaus, und die vermehrte Inanspruchnahme der Knochen führt an den Rändern der Wirbelkörper zu Wucher ungen, welche gegeneinander gerichtet sind und häufig miteinander verwachsen. Zu der Verdünnung der Bandscheiben kommt bei der Spondylitis deformans nicht selten Osteoporose der Wirbelkörper, die in einzelnen Fällen eine

1) Fortschr auf dem Gebiete der Röntgenstrahlen 1903, Bd. 7, S. 51 .

2) Festschrift zur 69. Versammlung der Naturforscher u. Ärzte. Braunschweig 1897. S. 109. 
Reduktion ihrer Höhe auf die Hälfte bewirkt (Simmonds). Es ist also verständlich, daß diese Erkrankungsform der Bandscheiben und Wirbelkörper in vorgeschrittenen Stadien zu erheblicher Verkürzung der Lendenwirbelsäule und entsprechendem Rippentiefstand führen kann; beträgt doch im Lendenteil die Höhe der Bandscheiben $1 / 3-1 / 2$ der Wirbelhöhe. Wie die Feststellung der Randwucherungen an den Wirbelkörpern im Röntgenbild als das sicherste Zeichen der Spondylitis deformans intra vitam anzusehen ist, so lassen auch die Röntgenstrahlen die Verdünnung der Bandscheiben an der Verschmälerung der Wirbelzwischenräume deutlich erkennen.

Wenn auch die Mehrzahl der neueren Autoren annimmt, daß die von Bechterew, Strümpell und Pierre Marie beschriebenen Typen der chronischen Wirbelsäulenversteifung sich weder anatomisch noch klinisch scharf voneinander trennen lassen und daß sie mehr quantitativ als qualitativ voneinander verschieden sind, so muß doch vom anatomischen Standpunkt neben der Spondylitis deformans die wohl charakterisierte, als Spondylitis ankylopoëtica bczeichnete Erkrankung hervorgehoben werden. Bei ihr handelt es sich bekanntlich um eine Erkrankung und Ankylosierung der kleinen Wirbelgelenke, die nach und nach die gesamte Wirbelsäule beteiligen und auch zu Verknöcherung der Bänder führen kann, während Bandscheiben und Wirbelkörper unbeeinflußt $z$ u bleiben pflegen. Aus diesem Grunde kommt es nicht zur Verkürzung der L.endenwirbelsäule und zum Rippentiefstand. Wohl aber kann die nicht selten dabei eintretende starke kyphotische Verkrümmung der Wirbelsäule zur Senkung des Brustkorbs geyen das Becken führen.

Für die klin is che Diagnose der Spondylitis deformans sind besonders die folgenden Punkte von Bedeutung. Die Patienten haben meist das mittlere Lebensalter überschritten. Die Wirbelsäule ist in der Regrel auffallend gerade, indem die dorsale Kyphose und die lumbale Lordose fehlen. Die Beweglichkeit der Wirbelsäule ist erheblich eingeschränkt, besonders bei Seitenbeugung des Rumpfes. In vorgeschrittenen Fällen besteht Rippentiefstand. Die Verkürzung der Lendenwirbelsäule führt zu wulstartigem Vortreten der Weichteile in der seitlichen Lendengegend, oder es treten hier mehr oder weniger tiefe Querfalten in der Haut auf. Subjektive Beschwerden können auch bei ausgebildeter Spondylitis deformans fehlen; sind sie vorhanden, so beziehen sie sich auf Erschwerung der Körperbewegungen sowie 
auf Schmerzen beim Bücken, Aufrichten und Heben. Außerdem können sich ausstrahlende Schmerzen im Gebiet der lumbalen und sakralen Nerven geltend machen. Wie Ischiasschmerzen häufig sekundär auf Arthritis deformans des Hüftgelcnks zu beziehen sind, so läßt sich für sie auch gelegentlich die Spondylitis deformans bzw. der durch diese bedingte Rippentiefstand ursächlich verantwortlich machen.

I) e page (Brit. med. Journ. 1908 II I004) hat in 4 Jahren 30 Fälle bei Frauen beobachtet, wo heftige Schmerzen in der rechten, seltener in der linken Seite bestanden, die fälschlich mit verschiedenen Organerkrankungen, am häufigsten mit Wanderniere oder Appendicitis, in Zusammenhang gebrache waren. Als Ursache der Beschwerden ergab sich Senkung der X. und XI. Rippe, welche den Beckenkamm berührten. Es handelte sich nicht um die bekannte Rippensenkung bei der „rachitischen Skoliose“, es fand sich jedoch oft ein leichter Grad von Skoliose. Viermal wurden die vorderen Rippenenden mit Erfolg reseziert. Ob es sich um Fälle von Spondylitis deformans oder um eine sonstige Ursache für den Rippentiefstand gehandelt hat, ergibt sich aus der Mitteilung Depages nicht.

Um über die Häufigkeit und die Ursachen des Rippentiefstandes ins klare zu kommen, habe ich bei über 300 Männem im Alter von 15 bis 75 Jahren, die beliebige Unfälle außerhalb der Wirbelsäule erlitten hatten oder sich wegen der verschiedenartigsten krankhaften Prozesse um die Invalidenrente bewarben, eine genaue Untersuchung der Wirbelsäule vorgenommen. Dabei stellte sich zunächst heraus, daß bei der männlichen arbeitenden Bevölkerung eine in bezug auf Gestalt, Länge und Beweglichkeit vollkommen normale Wirbelsäule selten ist, daß vielmehr schon im jugendlichen Alter, außer Abweichungen von den physiologischen Krümmungen, sowohl Rippentiefstand als Beweglichkeitsbeschränkung öfter beobachtet werden. Mit zunehmendem Alter wird die Zahl dieser krankhaften Veränderungen progressiv größer. Bei jugendlichen Personen konnte mehrfach $\mathrm{R}$ a chit is als Ursache des Rippentiefstandes beschuldigt werden. So hatten sich z. B. bei einem 18 jährigen jungen Mann, der. mit Coxa vara und Genu valgum behaftet war, die Rippen bis über die Beckenkämme hinaus gesenkt, bei abnorm gestreckter (gerader) 'Wirbelsäule. Ferner scheint es auch, daß 
frühzeitige starke Belastung, etwa durch fortgesetztes schweres Tragen, bei schwach entwickelten jugendlichen Individuen Verkürzung der Wirbelsäule und Rippentiefstand bewirken kann. Im vorgerückten Alter ist es die senile Atrophieder Band. scheiben und die Osteoporose der Wirbelkörper, welche unter dem Einfluß der Körperlast oder beruflicher Mehrbelastung Verkürzung der Wirbelsäule mit Rippentiefstand zur Folge haben können, ohne daß gleichzeitig die für die Spondylitis deformans charakteristischen Randwucherungen an den Wirbelkörpern aufzutreten brauchen. Man wird wohl voraussetzen dürfen, daß die Beweglichkeit der durch rein atrophischsenile Vorgänge verkürzten Wirbelsäule länger und besser erhalten bleibt als der durch Spondylitis deformans veränderten Wirbelsäule, obwohl bei dieser Erkrankung, selbst in ausgesprochenen Fällen, zuweilen die Beschränkung der Rumpfbewegungen auffallend gering ist.

In den von mir genauer untersuchten Fällen (ca. 300) waren in 1,7 Proz. die untersten Rippen in das Becken eingetreten, in 12 Proz. hatten sie sich bis zum Beckenkamm gesenkt, in I I,5 Proz. betrug der Abstand der I I. Rippe vom Beckenkamm I Fingerbreite, in je 29 Proz. Daumenbreite und 2 Fingerbreiten, in 12 Proz. 3 Fingerbreiten, in 4,7 Proz. 4 Fingerbreiten.

Wenn man nur einen Rippenabstand vom Beckenkamm von über 2 bis 4 Fingerbreiten als normal gelten lassen will 1 ), so würde sich demnach aus jenen Zahlen ergeben, daß von ca. 300, der arbeitenden Klasse angehörenden, in einem Alter zwischen 15 und 75 Jahren befindlichen Personen nur etwa I7 Proz. normalen Abstand zwischen II. Rippe und Beckenkamm aufwiesen. Wie sich die oben namhaft gemachten Ursachen des Rippentiefstandes auf die verschiedenen untersuchten Altersperioden verteilen, vermag ich nicht anzugeben. Betont sei jedoch nochmals, daß sich das Verhältnis der vorstehend als normal definierten Rippenabstände $z u$ den geringeren Abständen mit zunehmendem Alter stark zuungunsten der normalen verschob. Während nämlich bei den 15 - bis 40 jährigen die Fälle mit unter-

I) Eine gewisse physiologische Breite resultiert schon aus der schwankenden Dicke der normalen Bandscheiben. 
normalem Rippenabstand das Doppelte und 2- bis 4 fache der normalen betrugen, schwankten sie bei den höheren Altersstufen zwischen etwa dem 5- und 9 fachen; bei den über 65 Jahre alten Untersuchten fanden sich keine normalen Rippenabstände mehr.

Interessant ist es auch, das Höhenverhältnis $\mathrm{zwi}$ schen Beckenkamm und Epicondylus int. humeri bei stehenden Personen mit herabhängenden Armen zu verfolgen. Eine klare Gesetzmäßigkeit scheint hier nicht $z$ u herrschen, indem auch bei normal gebauten Personen die Verbindungslinie zwischen den beiderseitigen inneren Epicondylen des Humerus teils höher, teils tiefer als die Verbindungslinie der Beckenkämme zu stehen kommt, wobei besonders die Körpergröße von Einfluß zu sein scheint. Aber es läßt sich doch sagen, daß bei normalem Abstand der untersten Rippen vom Beckenkamm wesentlich häufiger die Epicondylenlinie oberhalb der Beckenkammlinie liegt oder mit ihr sich in gleicher Höhe befindet. Treten nun bei solchen Individuen Zustände ein, die starke Verkürzung der Lendenwirbelsäule und Rippentiefstand herbeiführen, so ist es begreiflich, daß dann das Höhenverhältnis der beiden fraglichen Linien eine Umkehrung erfahren muß, indem die Beckenlinie über die Epicondylenlinie hinaus nach oben rückt. So habe ich mehrere Fälle aus verschiedenen Altersperiodien untersucht, bei denen die Zeichen der Spondylitis deformans, als Rippentiefstand bis auf Daumenbreite und Querfaltenbildung, zusammenfielen mit Tieferstehen der Epicondylenlinie um 3 Fingerbreiten unterhalb der Beckenkammlinie, und wo vermutet werden konnte, daß dieses Verhältnis die Folge des Rippentiefstandes war.

Zusammenfassend läßt sich also sagen: Wenn der Abstand der I I. Rippe vom Beckenkamm in der Scapularlinie bei nicht unter mittelgroßen Personen nur 2 Fingerbreiten oder weniger beträgt, so deutet dies auf eine abnorme Verkürzung der Lendenwirbelsäule hin, die ihre Ursache in Höhenabnahme der durch Rachitis oder Osteoporose veränderten Wirbelkörper oder in Verdünnung der Bandscheiben infolge von Altersatrophie oder Degeneration bei Spondylitis deformans zu haben pflegt. Der Rippentiefstand stellt also an sich ein wichtiges, pathologische 
Veränderungen der Wirbelsäule anzeigendes Symptom dar, und seine Feststellung muß dazu auffordern, das übrige Verhalten der Wirbelsäule zu prüfen und zu ergründen, welche ursächlichen anatomischen Veränderungen vorliegen.

Da der Rippentiefstand bei Personen, die das mittlere Lebensalter überschritten haben, so häufig die Folge von senilatrophischen Prozessen an der Wirbelsäule oder von Spondylitis deformans ist, bedeutet sein Bestehen meist, daß körperliche $V$ erbrauchtheit vorliegt, und daß die allgemeine Arbeitsfähigkeit der Untersuchten herabgesetzt ist. Es ist deshalb wichtig, bei allen älteren B ewerbern um die Invalidenrente darauf zu untersuchen, ob Rippentiefstand vorliegt, was bejahendenfalls weiter zu einer genauen Feststellung der Gestalt und Beweglichkeit der Wirbelsäule führen muß. Wenn man sich einmal daran gewöhnt hat, bei den fraglichen Untersuchungen in dieser Weise vorzugehen, so erstaunt man über die Häufigkeit des Rippentiefstandes bei älteren, arbeitsbeschränkt oder arbeitsunfähig gewordenen Personen.

Aber auch in der Unfallchirurgie kann die Berücksichtigung des Abstandes zwischen untersten Rippen und Beckenkamm von Nutzen sein. Ungemein häufig sehen wir ja Fälle, wo Männer im mittleren Lebensalter nach scheinbar ganz unbedeutenden Quetschungen oder Stauchungen der Wirbelsäule langdauernde Beschwerden und Arbeitsunfähigkeit davontragen. Die Untersuchung ergibt dann recht oft abnorm gerade und stark bewegungsbeschränkte Wirbelsäule, Klopfschmerz der Dornfortsätze und Schmerzhaftigkeit der Bewegungen, ferner Rippentiefstand, Wulst- oder Faltenbildung in den Flanken und event. Hochstand der Epicondylenlinie im oben definierten Sinne. Kommt noch der Befund der charakteristischen Knochenwucherungen im Röntgenbild dazu, dann steht die Diagnose eines fortgeschrittenen Falles von Spondylitis deformans fest, und es ist nun die schwierige Frage zu entscheiden, inwieweit der erlittene Unfall für die bestehende Erkrankung bzw. für die Beschwerden verantwortlich gemacht werden kann. Nicht selten sind die Symptome der Spondylitis so stark ausgebildet, daß ihre Entstehung ohne weiteres in die Zeit vor dem Unfall verlegt werden muß. Aber auch sonst ist es wenig wahrscheinlich, daß ein so 
ausgesprochen chronisch-degenerativer Prozeß, wie die Spondylitis deformans, sich infolge von einmaligem Trauma aus einem normalen Zustand heraus entwickeln sollte. Wir brauchen aber auch nicht einmal Verschlimmerung oder Beschleunigung der vor dem Unfall schon vorhanden gewesenen Erkrankung der Wirbelsäule anzunehmen und können uns doch für Befriedigung der Rentenansprüche unserer Untersuchten aussprechen. Zunächst ist die Geringfügigkeit des Traumas, die so häufig den Verletzten in den Verdacht der Simulation bringt, bei bestehender Spondylitis deformans als Ursache intensiver Beschwerden nicht auffallend. Wissen wir doch, daß die Bandscheiben unter phy: siologischen Bedingungen gewissermaßen als Puffer wirken, die vermöge ihrer Elastizität die die Wirbelsäule treffenden Stöße und einseitig wirkenden Kräfte nach allen Richtungen innerhalb ihrer Substanz verbreiten und gleichmäßig auf die ganze Wirbelfläche verteilen, wodurch sie abgeschwächt und ausgeglichen werden. Es ist aber für die Spondylitis deformans in besonderem Grade bezeichnend, daß die degenerierten und er. weichten Bandscheiben ihre Elastizität vollkommen einbüßen, wodurch einerseits die Tragfähigkeit der Wirbelsäule beeinträchtigt wird, andererseits sie treffende Stöße unvermindert von Wirbelkörper zu Wirbelkörper vordringen können, was Rokitansky als die Hauptursache der Entwicklung der Spondylitis deformans ansah. Ist nun gar durch Verwachsung der Osteophyten eine Ankylose zustande gekommen, so wird eine so veränderte Wirbelsäule als besonders empfindlich gegen direkte und indirekte traumatische Einwirkungen angesehen werden können, d. h. die an Spondylitis deformans leidende Wirbelsäule wird mit Gewebsläsionen auch auf solche Traumen reagieren können, die wegen ihrer Geringfügigkeit eine normale Wirbelsäule nicht wesentlich zu beeinflussen vermögen. Bei den fraglichen Gewebsläsionen ist an während des Lebens nicht nachweisbare Einrisse der Bandscheiben, Fissuren oder Absprengungen an den osteoporotischen Knochen oder Kontinuitätstrennungen an den pathologischen Knochenwucherungen $\mathrm{zu}$ denken.

P la t $\mathrm{e}^{1}$ ) berichtet über einen Fall, bei dem heftige, in das rechte Bein ausstrahlende Schmerzen im Kreuz ganz plötzlich, blitzartig

I) Fortschr. auf d. Gebiete der Röntgenstrahlen I910/11, Bd. I6, S. 346. 
nach einer unvorsichtigen Bewegung entstanden waren. Wenige Wochen später fanden sich so starke Zeichen einer Spondylitis deformans, wie Brückenbildung, daß angenommen werden mußte, ihre Entstehung habe lange vor dem Trauma begonnen. Es wird als das wahrscheinlichste bezeichnet, daß anläßlich der Bewegung eine der Exostosen lädiert oder gebrochen war.

Wie oben bereits betont wurde, verläuft die Spondylitis deformans sehr oft ohne jede subjektiven Symptome; dies ist der hauptsächlichste Grund, warum ihre Häufigkeit unterschätzt zu werden pflegt. Es tritt eben meist weitgehende Anpassung an die anatomischen Veränderungen und die dadurch bedingten Funktionsstörungen ein. Diese Patienten befinden sich aber in einem labilen Gleichgewichtszustand, der durch traumatische Einflüsse aufgehoben werden kann, ohne daß jedesmal wesentliche anatomische Läsionen stattgefunden zu haben brauchen. Mit Recht hat man diese Verhältnisse verglichen mit derjenigen Form des traumatischen Plattfußes, bei der ein bis dahin symptomloser Plattfuß durch ein unter Umständen ganz leichtes Trauma insuffizient wird, $d$. h. zu eventuell langdauernden Beschwerden führt ( $\left.\mathrm{S} \mathrm{chanz} z^{1}\right)$ ).

Auf Grund vorstehender Erwägungen sind wir denn auch meistens in Fällen, wo bei bestehender, aber bis dahin symptomloser Spondylitis deformans ein relativ geringfügiges Trauma angeblich heftige und langdauernde Beschwerden mit entsprechender Beschränkung der Arbeitsfähigkeit herbeigeführt hat - vorausgesetzt, daß die betr. Klagen glaubhaft erscheinen-, berechtigt, für die Anerkennung der Rentenansprüche einzutreten.

Meine Erfahrungen veranlassen mich zu der Empfehlung, bei Untersuchung der Wirbelsäule die besondere Aufmerksamkeit darauf zu richten, ob Rippentiefstand vorhanden ist.

I) Berliner klin. Wochenschr. 1907, Nr. 3 I. 